\title{
Modifiable Risk Factors Explain Socioeconomic Inequalities in Dementia Risk: Evidence from a Population-Based Prospective Cohort Study
}

\author{
Kay Deckers ${ }^{\mathrm{a}, *}$, Dorina Cadar ${ }^{\mathrm{b}}$, Martin P.J. van Boxtel ${ }^{\mathrm{a}}$, Frans R.J. Verhey ${ }^{\mathrm{a}}$, Andrew Steptoe ${ }^{\mathrm{b}, \mathrm{c}}$ \\ and Sebastian Köhler ${ }^{\mathrm{a}}$ \\ ${ }^{a}$ Alzheimer Centrum Limburg, School for Mental Health and Neuroscience, Maastricht University, \\ Maastricht, the Netherlands \\ ${ }^{\mathrm{b}}$ Department of Behavioural Science and Health, University College London, London, UK \\ ${ }^{\mathrm{c}}$ Department of Epidemiology and Public Health, University College London, London, UK
}

Accepted 9 July 2019

\begin{abstract}
.
Background: Differences in dementia risk across the gradient of socioeconomic status (SES) exist, but their determinants are not well understood.

Objective: This study investigates whether health conditions and lifestyle-related risk factors explain the SES inequalities in dementia risk.

Methods: 6,346 participants from the English Longitudinal Study of Ageing were followed up from 2008/2009 until 2014/2015. We used Cox regression adjusted for age, gender, wealth/education, and clustering at the household level to examine the association between SES markers (wealth, education) and time to dementia in a structural equation model including potential mediation or effect modification by a weighted compound score of twelve modifiable risk and protective factors for dementia ('LIfestyle for BRAin health' (LIBRA) score).

Results: During a median follow-up of 6 years, 192 individuals $(3.0 \%)$ developed dementia. LIBRA scores decreased with increasing wealth and higher educational level. A one-point increase in the LIBRA score was associated with a $13 \%$ increase in dementia risk (hazard ratio $(\mathrm{HR})=1.13,95 \%$ confidence interval 1.07-1.19). Higher wealth was associated with a decreased dementia risk $(\mathrm{HR}=0.58,0.39-0.85)$. Mediation analysis showed that $52 \%$ of the risk difference between the highest and lowest wealth tertile was mediated by differences in LIBRA (indirect effect: HR $=0.75,0.66-0.85$ ). Education was not directly associated with dementia $(\mathrm{HR}=1.05,0.69-1.59)$, but was a distal risk factor for dementia by explaining differences in wealth and LIBRA scores (indirect effect high education: HR $=0.92,0.88-0.95$ ).

Conclusion: Socioeconomic differences in dementia risk can be partly explained by differences in modifiable health conditions and lifestyle factors.
\end{abstract}

Keywords: Aging, cohort study, dementia, epidemiology, health inequalities, lifestyle, mediation, prevention, public health, risk factors, socioeconomic status

\footnotetext{
*Correspondence to: Kay Deckers, PhD, Alzheimer Centrum Limburg, Department of Psychiatry and Neuropsychology, School for Mental Health and Neuroscience, Maastricht University, P.O. Box 616, 6200 MD Maastricht, the Netherlands. Tel.: +31 43 3884104; Fax: +31 43 3884092; E-mail: kay.deckers@ maastrichtuniversity.nl.
}

\section{INTRODUCTION}

Socioeconomic inequalities are a major contributor to the widening health gap, both within and between populations [1]. A recent multi-cohort study and meta-analysis of 1.7 million individuals showed 
that low socioeconomic status (SES) was associated with a decline of 2.1 years in life expectancy [2]. SES is a broad concept used to determine an individual's social standing and includes prestige- and resource-based measures. Prestige-based measures refer to an individual's relative status in the social hierarchy (e.g., parental educational level, an individual's position within an occupation classification system (e.g., the International Standard Classification of Occupations)), whereas resource-based measures include assets such as wealth, total family income and educational attainment [3].

Socioeconomic disparities have been well described for certain diseases, including different types of cancer and cardiovascular disease [4-6], but are less well understood in dementia. In a metaanalysis of almost 87,000 persons from the United Kingdom, a low educational level was associated with a $76 \%$ increased risk of dementia death in women [7]. Educational attainment is a marker of childhood SES, but is also strongly related to intelligence and level of cognitive activity, making it less suited as a 'pure' SES marker in dementia research [8]. In contrast, few studies used resource-based measures of SES. A prospective biracial cohort showed that both prestige- and resource-based measures of SES (educational level, literacy level, family income, and perceived financial inadequacy) contribute to disparities in dementia incidence among black and white participants [9], whereas another prospective study of older Mexican Americans showed an association between life-course socioeconomic position (e.g., parental educational level, lifetime occupation) and risk of cognitive impairment and dementia [10]. Recent results from the English Longitudinal Study of Ageing (ELSA) showed that wealth was associated with incident dementia even after controlling for education, while education was not an independent predictor of incident dementia [11].

Factors explaining SES inequalities in dementia risk are largely unknown and need further research [12], but include micro- (e.g., differences in risk factors exposure, health literacy, motivation), meso(e.g., social factors, the built environment), and macro-level (e.g., access to health care, income distribution, wider political forces) factors. Recent studies suggest that there is potential for dementia prevention through lifestyle adaptations, especially if started in midlife [13-19]. Differences in the distribution of modifiable dementia risk factors, treatable by cardiovascular risk management or lifestyle adaptations, might thus partly underlie the gap in dementia risk between poor and rich.

Therefore, this study aimed to investigate whether modifiable health conditions and lifestyle factors explain wealth inequalities in dementia incidence. In addition, we explored whether education might be part of this pathway.

\section{MATERIALS AND METHODS}

\section{Study design and participants}

ELSA is a multi-center panel study representing the English population aged 50 and over with bi-annual waves [20], collecting data on health, economics, lifestyle, psychology, and social wellbeing. More details have been published elsewhere [21].

For this study, Wave $4(2008 / 2009 ; n=9,886)$ was considered the baseline because it covered a large number of modifiable risk and protective factors for dementia. If possible, identical information from other waves (in most cases Wave 3 (2006/2007) or Wave 5 (2010/2011); Supplementary Table 1) was used if data on wealth, educational level and lifestyle factors were missing. The last assessment was Wave 7 (2014/15) yielding a maximum followup period of 7 years. After exclusion of (prevalent) dementia cases at Wave $4(n=209)$, and persons with fewer than eleven 'LIfestyle for BRAin health' (LIBRA) factors at Wave $4(n=1,374)$, loss to followup (e.g., removal, withdrawal, lost contact, moved abroad, death, etc.; $n=1,940$ ), invalid sampling weight $(n=12)$ or missing education data $(n=5)$, the analysis sample included 6,346 individuals.

The National Health Service Multicentre Research and Ethics Committee and the University College London Research Ethics Committee approved the study according to the guidelines of the Helsinki Declaration. All participants provided written informed consent.

\section{Measures}

\section{Dementia ascertainment}

The diagnosis of dementia was based on a combined algorithm of (1) physician-diagnosed dementia or Alzheimer's disease self-reported by the participant or their informant during the computer-assisted personal interview; or (2) an average score of $\geq 3.38$ on the shortened version of the Informant Question- 
naire on Cognitive Decline in the Elderly (IQCODE) [22-25]. This questionnaire uses informant reports to measure the change in cognitive abilities (e.g., memory) based on the pre-morbid level of functioning [26]. Each item was scored on a 1 (much improved) to 5 (much worse) range. The validity of this scale was previously examined, and the threshold used has both high specificity $(0.84)$ and sensitivity $(0.82)[25$, 27].

\section{Socioeconomic status indicators}

Self-reported household wealth was considered a resource-based indicator of SES at Wave 4, calculated by summing wealth from the total value of a respondent's home (minus outstanding mortgage payments), physical wealth (e.g., jewelry, artwork), business assets (e.g., investments), and financial assets such as cash and savings (minus debts and loans). The overall measure of wealth was divided into tertiles (low, medium, high).

Educational level was used as a prestige-based marker of SES at Wave 4. This variable indicating the highest level of education achieved was regrouped into three categories: low (no formal qualifications), medium (ordinary (O-) level or secondary education (equivalent to (junior) high school)) or high (advanced (A-) level or above (college/university)).

\section{Health- and lifestyle factors}

Health- and lifestyle factors were captured by a poly-environmental risk score called the LIBRA index [14]. LIBRA was developed after triangulation of results from a systematic literature review on risk and protective factors for dementia and an expert consensus study and consists of twelve modifiable risk and protective factors for dementia [14]. Risk factors are coronary heart disease, (type-2) diabetes, hypercholesterolemia, hypertension, depression, obesity, smoking, physical inactivity, and renal disease. Protective factors are low-to-moderate alcohol use, high cognitive activity and a healthy diet. A weight was assigned to each factor, based on the factor's relative risk [14]. Weights were then standardized and summed to yield the final LIBRA score (range: -5.9 to +12.7 ). Higher scores have been shown to predict cognitive decline and higher dementia risk in previous (population- and patient-based) cohort studies [28-31].

In ELSA, information was available for all LIBRA factors at Wave 4, except for renal dysfunction, based on clinical data from nurse visits or self- reported information (theoretical range from -5.9 to 11.6; Supplementary Table 2). Each measure was dichotomized according to established cut-offs (Supplementary Table 2) before assigning the weights.

\section{Statistical analyses}

Independent samples $t$-tests and $\chi^{2}$-tests tested differences in demographics, SES, and LIBRA factors between individuals with and without incident dementia. Structural equation modelling for continuous-time survival analysis (Cox proportional hazard regression) examined the associations between SES, LIBRA and time to dementia, resulting in hazard ratios (HR) and their $95 \%$ confidence interval (CI). The proportional hazard assumption was assessed based on the Schoenfeld residuals. To study mediation by lifestyle factors of the association between SES and incident dementia, the total effect of wealth was decomposed into direct and indirect (mediated) effects [32]. In all analyses, dementia was treated as the failure event. Survival time was used on the time axis and was calculated from the date of Wave 4 interview to date of dementia diagnosis (as reported by the participant or calculated as the midpoint between waves) or study exit (date of death or date of the last interview, whichever came first). Since participants could come from the same household, we used a sandwich estimator to allow clustering at the household level [33]. In addition, a sampling weight (baseline cross-sectional weight) was used in order to back-weight estimates from the analysis sample to the total sample to minimize selection bias. All analyses were adjusted for age, gender and wealth or education. Analyses were conducted in Stata 15.1 (StataCorp, College Station, TX, USA) and Mplus 8 (Muthén \& Muthén).

\section{RESULTS}

\section{Sample characteristics}

During the median follow-up of 6 years (interquartile range 0.33), 192 individuals $(3.0 \%)$ developed dementia (incidence rate $=52.0(95 \%$ CI, 45.0-60.4) per 10,000 person-years). The mean age was 64.9 years (standard deviation (SD) 8.6, range 50-94), and 3,536 (55.7\%) were female. Baseline characteristics are summarized by dementia status in Table 1. 
Table 1

Baseline characteristics of ELSA Wave 4 participants by incident dementia status

\begin{tabular}{|c|c|c|c|}
\hline \multirow[b]{2}{*}{ Variable } & \multicolumn{3}{|c|}{ Incident dementia status } \\
\hline & Yes $(n=192)$ & No $(n=6,154)$ & $p$ \\
\hline \multicolumn{4}{|l|}{ Demographics } \\
\hline Age, mean (SD) & $77.3(8.6)$ & $64.5(8.3)$ & $<0.001$ \\
\hline Female, $\mathrm{n}(\%)$ & $124(64.6)$ & $3,412(55.4)$ & 0.012 \\
\hline Educational level, n (\%) & & & $<0.001$ \\
\hline Low & $112(58.3)$ & $2,300(37.4)$ & \\
\hline Medium & $38(19.8)$ & $1,728(28.1)$ & \\
\hline High & $42(21.9)$ & $2,126(34.6)$ & \\
\hline Wealth, n (\%) & & & $<0.001$ \\
\hline Low & $84(43.8)$ & $1,798(29.2)$ & \\
\hline Medium & $57(29.7)$ & $2,133(34.7)$ & \\
\hline High & $51(26.6)$ & $2,223(36.1)$ & \\
\hline \multicolumn{4}{|l|}{ Health-and lifestyle factors } \\
\hline Heart disease, $\mathrm{n}(\%)$ & $41(21.4)$ & $538(8.7)$ & $<0.001$ \\
\hline Diabetes, n (\%) & $44(22.9)$ & $926(15.1)$ & 0.003 \\
\hline Hypercholesterolemia, n (\%) & $69(35.9)$ & $3,222(52.4)$ & $<0.001$ \\
\hline Hypertension, $\mathrm{n}(\%)$ & $86(44.8)$ & $1,979(32.2)$ & $<0.001$ \\
\hline Depression, n (\%) & $70(36.5)$ & $1,135(18.4)$ & $<0.001$ \\
\hline Obesity, n (\%) & $61(31.8)$ & $2,005(32.6)$ & 0.814 \\
\hline Smoking, n (\%) & $17(8.9)$ & $782(12.7)$ & 0.113 \\
\hline Low-to-moderate alcohol use, n (\%) & $72(37.5)$ & $3,508(57.0)$ & $<0.001$ \\
\hline Physical inactivity, n (\%) & $113(58.9)$ & $1,480(24.1)$ & $<0.001$ \\
\hline High cognitive activity, $\mathrm{n}(\%)$ & $33(17.2)$ & $2,747(44.6)$ & $<0.001$ \\
\hline Healthy diet, $\mathrm{n}(\%)$ & $105(54.7)$ & $3,687(59.9)$ & 0.146 \\
\hline LIBRA score, mean $(\mathrm{SD})^{*}$ & $1.9(2.8)$ & $-0.1(3.2)$ & $<0.001$ \\
\hline
\end{tabular}

$\mathrm{SD}$, standard deviation. LIBRA, LIfestyle for BRAin health; $p$ values are presented for the comparison of individuals with and without dementia at follow-up (independent samples $t$-tests and $\chi^{2}$-tests). ${ }^{*}$ LIBRA score theoretical range: -5.9 to 11.6 ; observed range: -5.9 to 10.3 .

\section{SES, lifestyle, and dementia}

LIBRA scores were lower in the highest wealth tertile $(n=2,274$; mean LIBRA score -1.37 , SD 2.79) in comparison with the lowest wealth tertile $(n=1,882$; mean LIBRA score 1.58, SD 3.13; $p<0.001$; Supplementary Figure 1), indicating higher risk. Likewise, individuals with medium $(n=1,766$; mean LIBRA score -0.18 , SD 3.06$)$ or high $(n=2,168$; mean LIBRA score -1.35, SD 2.87) education had lower LIBRA scores in comparison with individuals with low education $(n=2,412$; mean LIBRA score 1.18 , SD 3.05); $p<0.001)$. The incidence of dementia, was $77.0(62.0-96.8), 45.2(34.8-59.8)$, and 36.1 (27.3-48.7) per 10,000 person-years in the lowest, medium and highest wealth tertile, respectively. In line with previous findings, education was not associated with dementia risk (high education: $\mathrm{HR}=1.05$, 0.69-1.59). A one-point increase in LIBRA was, on average, associated with a $13 \%$ increase in dementia risk $(\mathrm{HR}=1.13,1.07-1.19$; Fig. 1). The association between LIBRA and dementia risk was stronger for people aged $50-70$ years $(\mathrm{HR}=1.20,1.06-1.36)$ than for those aged 70 years and older: $\mathrm{HR}=1.11$,

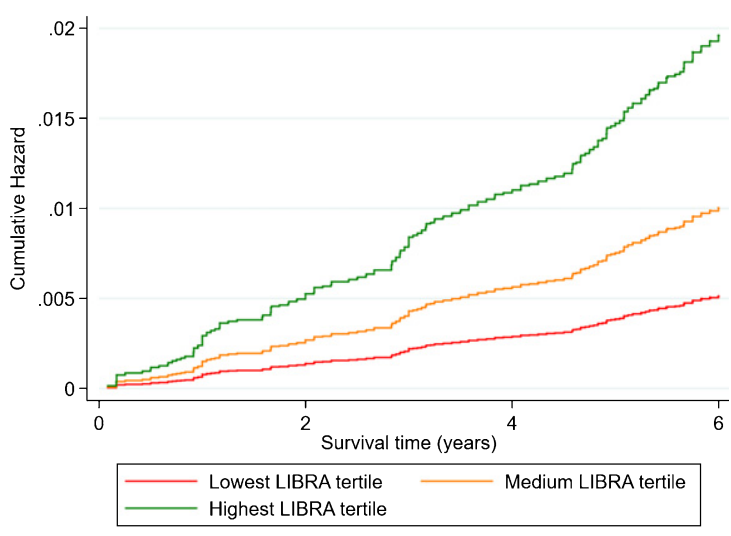

Model adjusted for age, gender, education, and clustering at the household level LIBRA, LIfestyle for BRAin health

Fig. 1. The cumulative hazard of dementia over time in study by LIBRA score tertiles.

1.05-1.17). Higher wealth was associated with a decreased risk of dementia (total effect; highest versus lowest wealth tertile: $\mathrm{HR}=0.58,0.39-0.85$; Fig. 2). 


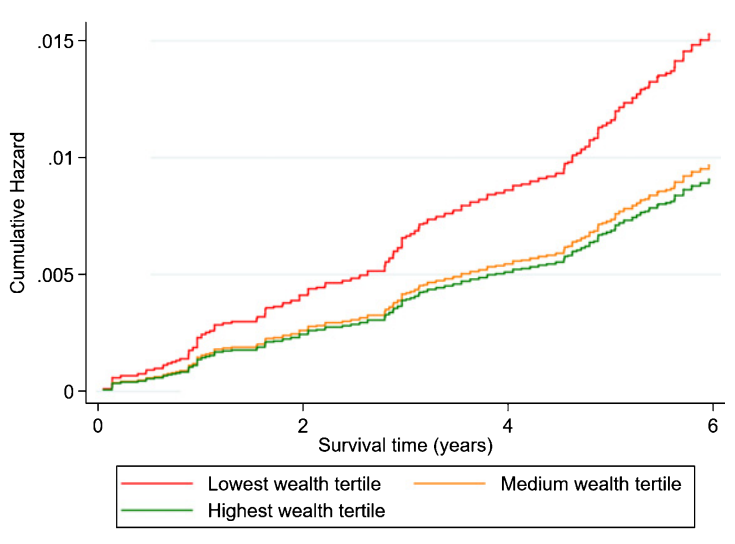

Model adjusted for age, gender, education, and clustering at the household level

Fig. 2. The cumulative hazard of dementia over time in study by wealth tertiles.

\section{Mediation analysis}

LIBRA significantly mediated the association between wealth and incident dementia (Fig. 3). Mediation analysis showed an effect for the indirect path from wealth to dementia via LIBRA (highest versus lowest wealth tertile: HR $=0.75,0.66-0.85$ ). Further calculations showed that differences in lifestyle factors explained $52.2 \%$ of the difference between the highest and lowest wealth tertile on dementia risk. There was no interaction of LIBRA with wealth, suggesting that lifestyle was similarly related to dementia risk across wealth strata. In contrast, there was no direct (residual) effect of wealth on dementia (highest versus lowest wealth tertile: $\mathrm{HR}=0.77,0.51-1.16$ ). Stronger but directionally similar mediation results were found in those aged 50 to 69 years (indirect path of wealth on dementia via LIBRA (lowest versus highest tertile): $\mathrm{HR}=0.64,0.47-0.86$ ) than those aged $\geq 70$ years $(\mathrm{HR}=0.81,0.72-0.91)$. Additional analyses for reversed causation did not show an association between LIBRA and dementia via wealth. That is, while there was a direct effect of LIBRA on wealth, there was no direct effect of wealth on dementia and no indirect effect of LIBRA on dementia via wealth.

\section{Testing the role of education as a distal risk factor}

While education was not associated with dementia risk once wealth was adjusted for (Supplementary Figure 2), we tested whether it is a distal risk factor via its associations with wealth and LIBRA. Mediation analysis showed that education indeed had no direct effect on dementia risk. However, high education was associated with higher wealth. In addition, there was an indirect path from education to dementia risk via wealth and LIBRA scores (indirect effect medium education: $\mathrm{HR}=0.96,0.94-0.98$; indirect effect high education: $\mathrm{HR}=0.92,0.88-0.95$; Supplementary Figure 3). Additionally, there was an indirect path from education to dementia via LIBRA (indirect effect medium education: $\mathrm{HR}=0.89,0.85-0.94$; indirect effect high education: $\mathrm{HR}=0.80,0.73-0.88$; Supplementary Figure 3). Taken together, higher education related to a lower dementia risk by its direct association with better lifestyle on the one hand, and by its association with higher midlife SES that in turns is associated with better lifestyle on the other hand.

\section{Sensitivity analyses}

The group with less than eleven LIBRA factors $(n=1,374)$ was on average older, with more dementia cases, (192/6,346 versus 52/588), less educated, had lower wealth, had higher number of health conditions (excluding obesity, hypercholesterolemia and hypertension) and lifestyle factors (excluding healthy diet). Those lost to follow-up $(n=1,940)$ were on average older, were more often male, were less educated, had lower wealth and had higher number of health conditions (excluding obesity) and lifestyle factors. Multiple imputation was used to impute missing values for the eleven LIBRA factors and educational level. Multivariate imputation by chained equations was carried out using all non-missing data on risk and protective factors and socio-demographic covariates (age, sex, and wealth) [34]. Ten imputed datasets were created, and the results combined using Rubin's rules [35]. After multiple imputation, the analysis sample consisted of 6,920 individuals, of which 238 (3.4\%) developed incident dementia. In line with the primary Cox proportional hazard regression analyses adjusted for age, gender, and wealth or education (and taking clustering at the household level into account), a one-point increase in LIBRA was, on average, associated with a $15 \%$ increase in dementia risk $(\mathrm{HR}=1.15,1.10-1.20)$, education was not associated with dementia risk (medium education: $\mathrm{HR}=0.76,0.53-1.10$; high education: $\mathrm{HR}=0.85$, $0.58-1.25$; in comparison with low education), and higher wealth was associated with a decreased risk of dementia (medium wealth: $\mathrm{HR}=0.64,0.46-0.90$; high wealth: $\mathrm{HR}=0.69,0.48-0.98$; in comparison with low wealth). 


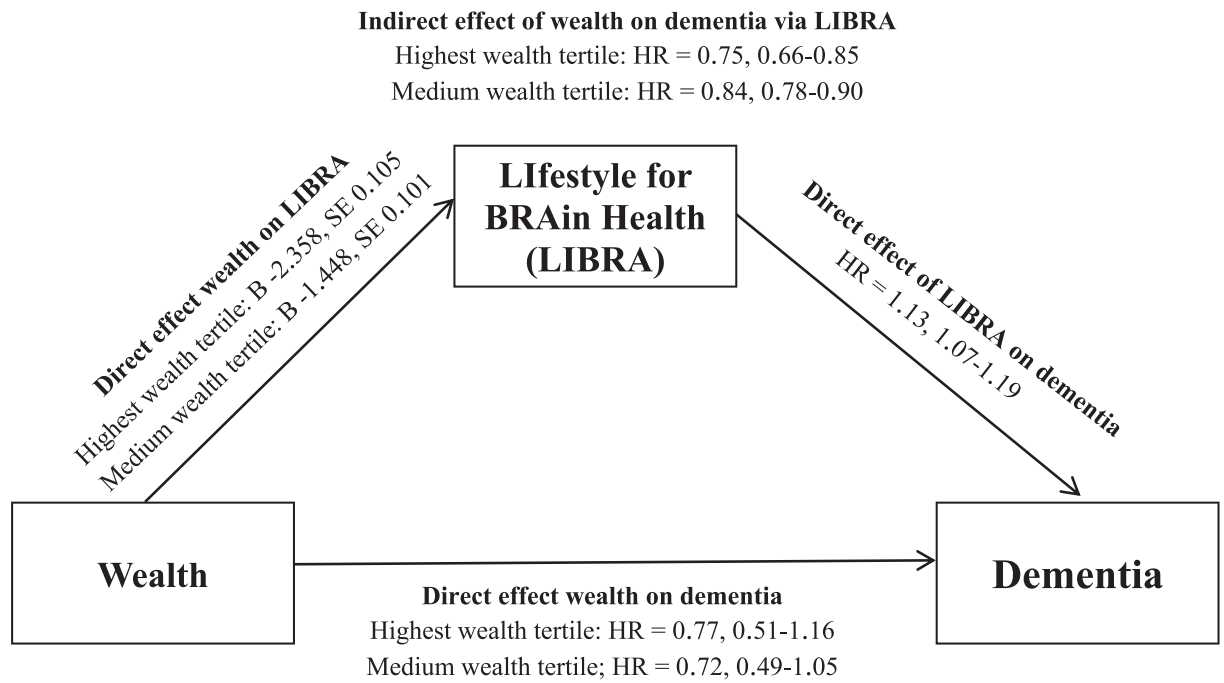

Fig. 3. Mediation analysis for the relationship between wealth and dementia as mediated by LIBRA. $B$, unstandardized regression coefficient; HR, hazard ratio; SE, standard error.

\section{DISCUSSION}

In this prospective cohort study, participants with lower wealth had a higher risk for dementia, which was to a large degree due to modifiable risk factors. In contrast, higher education had no direct effect on dementia risk, but showed lower risk by its relationship with higher wealth and better lifestyle/health. These findings suggest that improving the health and managing risk in those with both less wealth and low educational level might have an effect on dementia prevention by narrowing the gap in dementia risk between poor and rich.

The observed association between a less healthy lifestyle and dementia risk are in line with the results from previous studies that found an association between LIBRA measured in midlife and late life and incident dementia [30, 31]. In line with the few other studies that have examined the association between SES and dementia-related outcomes [7, 9, 10], we found that higher wealth, as a resource-based indicator of SES, was associated with decreased dementia risk. Only a few studies investigated the exact mechanisms underlying this association. A retrospective case-control study from Japan showed that diabetes minimally mediated the association between SES and dementia [36]. In contrast, we found mediation, in which more than $50 \%$ of the differences in dementia risk between the low and high wealth groups could be attributed to modifiable health conditions and lifestyle factors.
Low SES has been identified as one of the key barriers in facilitating healthy behavior in midlife in order to prevent dementia in late-life [37]. Several mechanisms can account for this observation. Low SES has been associated with low health literacy [38], less access to health resources and proper medical care [39], poor health behaviors and health outcomes [38, 40-44], of which some are related to increased risk of cognitive decline or dementia [14, 18, 45]. Higher education, a prestige-based indicator of high SES [8], is associated with better health choices and mixed results for dementia risk [11, 39, 46]. In line with recent research in ELSA [11], education was not directly associated with dementia risk. This seems in line with results from the Whitehall II study, which showed that low midlife occupation rather than low childhood education was associated with increased dementia risk [47]. Using mediation analysis, we were able to show that education is a distal risk factor for dementia in our sample that explains differences in wealth and lifestyle factors. Hence, strategies to improve access to (higher) education might also reduce dementia risk, especially in resource poor settings where education is not a universal good [18]. However, educational attainment also reflects inborn intelligence and predisposes individuals differently to engagement in stimulating mental activities during the life-course, thereby building up cognitive reserve that acts in return as a buffer against dementia risk [48]. Through this confounding, it might be a suboptimal indicator of SES in dementia research. 
Because uptake of advice and health recommendations tends to be greater among more educated and affluent people, one of the main challenges of behavior change programs to mitigate dementia risk will be to design them in order to reduce rather than increase social inequalities [49]. Additionally, focusing on the individual level alone might lead to excessive victimblaming. A strong common political focus is needed, including a national and global policy on the promotion of healthy brain behavior. In line with this, health services should be available for all layers of society based on an individual's need (universal proportionalism) [50].

Our study has several strengths, including an extended follow-up period in a large representative sample of the British population. Some important limitations have to be considered, too. First, participants with missing data (34\% of the total sample) were excluded from the analysis. Exclusion of these individuals has probably led to an underestimation of the observed associations. Our sensitivity analyses, including multiple imputation showed similar results as the primary analyses. Second, as an inevitable part of all longitudinal studies, not all participants who entered the study returned for the follow-up measurements. This might have led to some selection of a healthier sample and therefore may result in an underestimation of the "true" association. Third, the ascertainment of dementia diagnosis, wealth, and some LIBRA factors was based on self-report, which could have led to response bias or non-differential exposure misclassification. In order to reduce these forms of bias, we only included self-reported information when objective measurements (e.g., from nurse visits) were not available. Worldwide, dementia is underdiagnosed in the general population [51]. So, physician diagnoses (even if they are accurately reported) will underestimate dementia incidence. Fourth, possible interactions between risk and protective factors were not taken into account in the LIBRA score. Fifth, the observed associations may have been confounded by factors like childhood/parental SES, occupational experiences and SES mobility.

Despite these limitations, our study does have important implications and provides valuable input for public health policies. Targeting lifestyle factors in tailored health education programs might eventually lead to a reduction in socioeconomic inequalities in (brain) health. Effective public health programs in dementia prevention and care must take the needs and resources of this vulnerable group into consideration before deciding on the most appropriate preventa- tive measures at the individual, societal, and political level.

In conclusion, socioeconomic differences in dementia risk can be partly explained by differences in modifiable health conditions and lifestyle factors.

\section{ACKNOWLEDGMENTS}

This work was supported by a fellowship (Kay Deckers) from Alzheimer Nederland and the Alzheimer's Society UK [grant number WE.152015-01]. The English Longitudinal Study of Ageing is supported by the National Institute on Aging [grant numbers 2RO1AG7644-01A1，2RO1AG017644] and a consortium of the UK government departments coordinated by the Economic and Social Research Council.

Authors' disclosures available online (https:// www.j-alz.com/manuscript-disclosures/19-0541r2).

\section{SUPPLEMENTARY MATERIAL}

The supplementary material is available in the electronic version of this article: http://dx.doi.org/ 10.3233/JAD-190541.

\section{REFERENCES}

[1] Marmot M (2005) Social determinants of health inequalities. Lancet 365, 1099-1104.

[2] Stringhini S, Carmeli C, Jokela M, Avendano M, Muennig P, Guida F, Ricceri F, d'Errico A, Barros H, Bochud M, Chadeau-Hyam M, Clavel-Chapelon F, Costa G, Delpierre C, Fraga S, Goldberg M, Giles GG, Krogh V, Kelly-Irving M, Layte R, Lasserre AM, Marmot MG, Preisig M, Shipley MJ, Vollenweider P, Zins M, Kawachi I, Steptoe A, Mackenbach JP, Vineis P, Kivimaki M (2017) Socioeconomic status and the $25 \times 25$ risk factors as determinants of premature mortality: A multicohort study and meta-analysis of 1.7 million men and women. Lancet 389, 1229-1237.

[3] Krieger N, Williams DR, Moss NE (1997) Measuring social class in US public health research: Concepts, methodologies, and guidelines. Annu Rev Public Health 18, 341-378.

[4] Clark AM, DesMeules M, Luo W, Duncan AS, Wielgosz A (2009) Socioeconomic status and cardiovascular disease: Risks and implications for care. Nat Rev Cardiol 6, 712-722.

[5] Marmot MG, Bosma H, Hemingway H, Brunner E, Stansfeld S (1997) Contribution of job control and other risk factors to social variations in coronary heart disease incidence. Lancet 350, 235-239.

[6] Stringhini S, Dugravot A, Kivimaki M, Shipley M, Zins M, Goldberg M, Ferrie JE, Singh-Manoux A (2011) Do different measures of early life socioeconomic circumstances predict adult mortality? Evidence from the British Whitehall II and French GAZEL studies. J Epidemiol Community Health 65, 1097-1103.

[7] Russ TC, Stamatakis E, Hamer M, Starr JM, Kivimaki M, Batty GD (2013) Socioeconomic status as a risk factor for 
dementia death: Individual participant meta-analysis of 86 508 men and women from the UK. Br J Psychiatry 203, 10-17.

[8] Galobardes B, Shaw M, Lawlor DA, Lynch JW, Davey Smith G (2006) Indicators of socioeconomic position (part 1). J Epidemiol Community Health 60, 7-12.

[9] Yaffe K, Falvey C, Harris TB, Newman A, Satterfield S, Koster A, Ayonayon H, Simonsick E (2013) Effect of socioeconomic disparities on incidence of dementia among biracial older adults: Prospective study. BMJ 347, f7051.

[10] Zeki Al Hazzouri A, Haan MN, Kalbfleisch JD, Galea S, Lisabeth LD, Aiello AE (2011) Life-course socioeconomic position and incidence of dementia and cognitive impairment without dementia in older Mexican Americans: Results from the Sacramento area Latino study on aging. Am J Epidemiol 173, 1148-1158.

[11] Cadar D, Lassale C, Davies H, Llewellyn DJ, Batty G, Steptoe A (2018) Individual and area-based socioeconomic factors associated with dementia incidence in England: Evidence from a 12-year follow-up in the English Longitudinal Study of Ageing. JAMA Psychiatry 75, 723-732.

[12] Lincoln P, Fenton K, Alessi C, Prince M, Brayne C, Wortmann M, Patel K, Deanfield J, Mwatsama M (2014) The Blackfriars Consensus on brain health and dementia. Lancet 383, 1805-1806.

[13] Baumgart M, Snyder HM, Carrillo MC, Fazio S, Kim H, Johns H (2015) Summary of the evidence on modifiable risk factors for cognitive decline and dementia: A populationbased perspective. Alzheimers Dement 11, 718-726.

[14] Deckers K, van Boxtel MP, Schiepers OJ, de Vugt M, Munoz Sanchez JL, Anstey KJ, Brayne C, Dartigues JF, Engedal K, Kivipelto M, Ritchie K, Starr JM, Yaffe K, Irving K, Verhey FR, Kohler S (2015) Target risk factors for dementia prevention: A systematic review and Delphi consensus study on the evidence from observational studies. Int J Geriatr Psychiatry 30, 234-246.

[15] Ferri CP, Prince M, Brayne C, Brodaty H, Fratiglioni L, Ganguli M, Hall K, Hasegawa K, Hendrie H, Huang Y, Jorm A, Mathers C, Menezes PR, Rimmer E, Scazufca M (2005) Global prevalence of dementia: A Delphi consensus study. Lancet 366, 2112-2117.

[16] Livingston G, Sommerlad A, Orgeta V, Costafreda SG, Huntley J, Ames D, Ballard C, Banerjee S, Burns A, CohenMansfield J, Cooper C, Fox N, Gitlin LN, Howard R, Kales HC, Larson EB, Ritchie K, Rockwood K, Sampson EL, Samus Q, Schneider LS, Selbaek G, Teri L, Mukadam N (2017) Dementia prevention, intervention, and care. Lancet 390, 2673-2734.

[17] Ngandu T, Lehtisalo J, Solomon A, Levalahti E, Ahtiluoto S, Antikainen R, Backman L, Hanninen T, Jula A, Laatikainen T, Lindstrom J, Mangialasche F, Paajanen T, Pajala S, Peltonen M, Rauramaa R, Stigsdotter-Neely A, Strandberg T, Tuomilehto J, Soininen H, Kivipelto M (2015) A 2 year multidomain intervention of diet, exercise, cognitive training, and vascular risk monitoring versus control to prevent cognitive decline in at-risk elderly people (FINGER): A randomised controlled trial. Lancet 385, 2255-2263.

[18] Norton S, Matthews FE, Barnes DE, Yaffe K, Brayne C (2014) Potential for primary prevention of Alzheimer's disease: An analysis of population-based data. Lancet Neurol 13, 788-794.

[19] Prince M, Bryce R, Albanese E, Wimo A, Ribeiro W, Ferri CP (2013) The global prevalence of dementia: A systematic review and metaanalysis. Alzheimers Dement 9, 63-75 e62.
[20] Steptoe A, Breeze E, Banks J, Nazroo J (2013) Cohort profile: The English longitudinal study of ageing. Int J Epidemiol 42, 1640-1648.

[21] Rogers NT, Banks J, Nazroo J, Steptoe A (2016) English Longitudinal Study of Ageing (ELSA). In Encyclopedia of Geropsychology, Pachana AN, ed. Springer Singapore, Singapore, pp. 1-10.

[22] Jorm AF (1994) A short form of the Informant Questionnaire on Cognitive Decline in the Elderly (IQCODE): Development and cross-validation. Psychol Med 24, 145153.

[23] Jorm AF (2004) The Informant Questionnaire on cognitive decline in the elderly (IQCODE): A review. Int Psychogeriatr 16, 275-293.

[24] Jorm AF, Jacomb PA (1989) The Informant Questionnaire on Cognitive Decline in the Elderly (IQCODE): Socio-demographic correlates, reliability, validity and some norms. Psychol Med 19, 1015-1022.

[25] Quinn TJ, Fearon P, Noel-Storr AH, Young C, McShane R, Stott DJ (2014) Informant Questionnaire on Cognitive Decline in the Elderly (IQCODE) for the diagnosis of dementia within community dwelling populations. Cochrane Database Syst Rev, CD010079.

[26] Jorm AF, Korten AE (1988) Assessment of cognitive decline in the elderly by informant interview. Br J Psychiatry 152, 209-213.

[27] Jorm AF, Masaki KH, Davis DG, Hardman J, Nelson J, Markesbery WR, Petrovitch H, Ross GW, White LR (2004) Memory complaints in nondemented men predict future pathologic diagnosis of Alzheimer disease. Neurology 63, 1960-1961.

[28] Deckers K, Nooyens A, van Boxtel M, Verhey F, Verschuren M, Kohler S (2019) Gender and educational differences in the association between lifestyle and cognitive decline over 10 years: The Doetinchem Cohort Study. J Alzheimers Dis 70(Suppl 1), S31-S41.

[29] Pons A, LaMonica HM, Mowszowski L, Kohler S, Deckers K, Naismith SL (2018) Utility of the LIBRA Index in relation to cognitive functioning in a clinical health seeking sample. J Alzheimers Dis 62, 373-384.

[30] Schiepers OJ, Kohler S, Deckers K, Irving K, O’Donnell CA, van den Akker M, Verhey FR, Vos SJ, de Vugt ME, van Boxtel MP (2018) Lifestyle for Brain Health (LIBRA): A new model for dementia prevention. Int J Geriatr Psychiatry 33, 167-175.

[31] Vos SJB, van Boxtel MPJ, Schiepers OJG, Deckers K, de Vugt M, Carriere I, Dartigues JF, Peres K, Artero S, Ritchie K, Galluzzo L, Scafato E, Frisoni GB, Huisman M, Comijs HC, Sacuiu SF, Skoog I, Irving K, O'Donnell CA, Verhey FRJ, Visser PJ, Kohler S (2017) Modifiable risk factors for prevention of dementia in midlife, late life and the oldestold: Validation of the LIBRA Index. J Alzheimers Dis 58, 537-547.

[32] Pratschke J, Haase T, Comber H, Sharp L, de Camargo Cancela M, Johnson H (2016) Mechanisms and mediation in survival analysis: Towards an integrated analytical framework. BMC Med Res Methodol 16, 27.

[33] Williams RL (2000) A note on robust variance estimation for cluster-correlated data. Biometrics 56, 645-646.

[34] White IR, Royston P, Wood AM (2011) Multiple imputation using chained equations: Issues and guidance for practice. Stat Med 30, 377-399.

[35] Rubin DB (1996) Multiple imputation after 18+years. J Am Stat Assoc 91, 473-489. 
[36] Nakahori N, Sekine M, Yamada M, Tatsuse T, Kido H, Suzuki M (2018) A pathway from low socioeconomic status to dementia in Japan: Results from the Toyama dementia survey. BMC Geriatr 18, 102.

[37] Kelly S, Martin S, Kuhn I, Cowan A, Brayne C, Lafortune $\mathrm{L}$ (2016) Barriers and facilitators to the uptake and maintenance of healthy behaviours by people at mid-life: A rapid systematic review. PLoS One 11, e0145074.

[38] Berkman ND, Sheridan SL, Donahue KE, Halpern DJ, Crotty K (2011) Low health literacy and health outcomes: An updated systematic review. Ann Intern Med 155, 97-107.

[39] National Center for Health Statistics (2012) Health, United States, 2011: With Special Feature on Socioeconomic Status and Health, Hyattsville, MD, USA.

[40] Davey Smith G, Neaton JD, Wentworth D, Stamler R, Stamler J (1998) Mortality differences between black and white men in the USA: Contribution of income and other risk factors among men screened for the MRFIT. MRFIT Research Group. Multiple Risk Factor Intervention Trial. Lancet 351, 934-939.

[41] Krishnan S, Cozier YC, Rosenberg L, Palmer JR (2010) Socioeconomic status and incidence of type 2 diabetes: Results from the Black Women's Health Study. Am J Epidemiol 171, 564-570.

[42] Marmot MG, Smith GD, Stansfeld S, Patel C, North F, Head J, White I, Brunner E, Feeney A (1991) Health inequalities among British civil servants: The Whitehall II study. Lancet 337, 1387-1393.

[43] Marshall IJ, Wang Y, Crichton S, McKevitt C, Rudd AG, Wolfe CD (2015) The effects of socioeconomic status on stroke risk and outcomes. Lancet Neurol 14, 1206-1218.
[44] Smith GD, Hart C, Blane D, Hole D (1998) Adverse socioeconomic conditions in childhood and cause specific adult mortality: Prospective observational study. BMJ 316, 16311635.

[45] Plassman BL, Williams JW Jr, Burke JR, Holsinger T, Benjamin S (2010) Systematic review: Factors associated with risk for and possible prevention of cognitive decline in later life. Ann Intern Med 153, 182-193.

[46] Sharp ES, Gatz M (2011) Relationship between education and dementia: An updated systematic review. Alzheimer Dis Assoc Disord 25, 289-304.

[47] Rusmaully J, Dugravot A, Moatti JP, Marmot MG, Elbaz A, Kivimaki M, Sabia S, Singh-Manoux A (2017) Contribution of cognitive performance and cognitive decline to associations between socioeconomic factors and dementia: A cohort study. PLoS Med 14, e1002334.

[48] Meng X, D’Arcy C (2012) Education and dementia in the context of the cognitive reserve hypothesis: A systematic review with meta-analyses and qualitative analyses. PLoS One 7, e38268.

[49] Adler NE, Glymour M, Fielding J (2016) Addressing social determinants of health and health inequalities. JAMA 316, 1641-1642.

[50] Marmot M, Bell R (2012) Fair society, healthy lives. Public Health 126(Suppl 1), S4-10.

[51] Lang L, Clifford A, Wei L, Zhang D, Leung D, Augustine G, Danat IM, Zhou W, Copeland JR, Anstey KJ, Chen R (2017) Prevalence and determinants of undetected dementia in the community: A systematic literature review and a metaanalysis. BMJ Open 7, e011146. 